\title{
Fluorescence Counter-Staining of Cell Nuclear DNA for Multi-Color Laser Confocal Microscopy
}

\author{
Takeshi Suzuki, Toshiyuki Matsuzaki and Kuniaki Takata \\ Department of Cell Biology, Institute for Molecular and Cellular Regulation, Gunma University, Gunma 371-8512
}

Received for publication December 26, 1997

Twenty-one nucleic acid-specific fluorochromes were examined for use in cell nuclear counter-staining of specimens observed under a laser confocal microscope equipped with a Krypton/argon laser tube. Six green, three red, and two far-red fluorochromes gave sufficient fluorescence. Among them, SYBR Green I, Pico Green, SYTOX Green, YO-PRO-3, and TO-PRO-3 specifically stained the cell nuclear DNA. Other fluorochromes except POPO-3 stained the cell nuclear DNA and co-stained the cytoplasmic RNA. Conversely, POPO-3 specifically stained the cytoplasmic and nucleolar RNA. Anti-bleaching reagents (PPDA and
DABCO) effectively prevented the photobleaching of SYBR Green I, Pico Green, YO-PRO-3, and TO-PRO-3, whereas DABCO accelerated the photobleaching of SYTOX Green. Taking into account the characteristics of these fluorochromes, SYBR Green I, YO-PRO-3, and TO-PRO-3 were best suited for cell nuclear counter-staining as green, red, and far-red fluorochromes, respectively. Especially, TO-PRO-3 seems to be the most useful because its far-red fluorescence enables three-color fluorescence staining by combination with immunofluorescence labeling with fluorescein and rhodamine derivatives.

Key words: Nucleic acid-specific fluorochrome, Cell nuclei, Counter-staining, Anti-bleaching reagent, Laser confocal microscopy

\section{Introduction}

Fluorescence microscopy, including immunofluorescence methods, has been widely used in the observation and analysis of localization and behavior of cellular molecules. Moreover, three-dimensional analysis of these molecules has been made possible even in living cells by the introduction of laser confocal microscopy without laborious serial-sectioning of fixed specimens [29]. In fluorescence histochemistry, however, only fluorescence representing a particular molecule is visualized; other information such as cellular structure and shape of nuclei are usually obtained by fluorescence counter-staining or bright-field microscopy, i.e., phase contrast or differential interference-contrast microscopy. Since a bright-field image is not confocal, comparison between a conventional bright-field image and an optical section image obtained with a laser confocal microscope is prone to result in an erroneous interpretation of the images. Therefore, the

Correspondence to: Takeshi Suzuki, Ph. D., Department of Cell Biology, Institute for Molecular and Cellular Regulation, Gunma University, Showa-machi 3-39-15, Maebashi, Gunma 371-8512, Japan. fluorescence counter-staining for laser confocal microscopy is of great importance. We have stained F-actin and DNA with fluorescent-labeled phalloidin and DAPI (4',6-diamidino-2-phenylindole), respectively, for counter-staining to distinguish the cell contour and cell nucleus $[25,26]$. Recently, we examined nucleic acid binding by fluorescent dyes for use in nuclear counterstaining $[18,24]$. In this present communication we summarize and evaluate their usefulness in immunofluorescence labeling by laser confocal microscopy.

\section{Conventional Fluorochromes for Cell Nuclear Counter-Staining}

DAPI, a DNA-specific fluorochrome, has been widely used for counter-staining of cell nuclear DNA in conventional immunofluorescence microscopy [25]. DAPI is highly specific for DNA and emits a bright blue-white fluorescence under ultraviolet (UV) excitation. Only a small number of wavelengths, the length of which depend on the type of laser tubes, can be utilized for excitation in laser confocal microscopy compared with the greater number available for conventional fluorescence microscopy, which utilizes a wide spectral range from a high- 
pressure mercury bulb (Table 1) [1]. In addition, most laser confocal microscopes are not able to provide UV excitation, since an expensive large-scale, water-cooled, argon-laser system is required for UV excitation. The use of DAPI, therefore, is restricted to such a specialized system. Propidium iodide (PI), which binds to both DNA and RNA and emits red fluorescence under blue-green excitation (peak of excitation spectrum $=530 \mathrm{~nm}$ ), has widely been used instead for laser confocal microscopy [11-15, $19,21,22,24]$. However, PI is also excited by blue laser $(488 \mathrm{~nm})$, which wavelength is generally used for eliciting green fluorescence such as FITC fluorescence, and intense fluorescence of PI sometimes bleeds through the emission filter for green fluorescence.

\section{Novel Nucleic Acid-Specific Fluorochromes for Cell Nuclear Counter-Staining}

Recently, a variety of nucleic acid-binding fluorescent

Table 1. Laser tubes and their capable excitation wavelength (nm).

\begin{tabular}{lcccc}
\hline & ultraviolet & blue & green & red \\
\hline argon & - & 457,488 & 514 & - \\
water-cooled argon & 351,363 & 488 & 514 & - \\
krypton/argon & - & 488 & 568 & 647 \\
green-helium/neon & - & - & 543 & - \\
red-helium/neon & - & - & - & 633 \\
helium/cadmium & - & 442 & - & - \\
\hline
\end{tabular}

dyes have been developed [8] and have come into practical use in flow cytometry and in histochemical analysis $[2-7,9$, $10,16,17,20,27]$. We previously screened twenty-one nucleic acid-specific fluorescent dyes (POPO-1, PO-PRO1, BOBO-1, BO-PRO-1, YOYO-1, YO-PRO-1, SYBR Green I, Pico Green, SYTOX Green, TOTO-1, TO-PRO1, POPO-3, PI, ethidium homodimer-2, PO-PRO-3, BOBO-3, BO-PRO-3, YOYO-3, YO-PRO-3, TOTO-3, and TO-PRO-3) for use in cell nuclear counter-staining in conjunction with laser confocal microscopy [18, 24]. With a laser confocal microscope (MRC-1024; Bio-Rad Laboratories, Hercules, CA) equipped with a 15-mW krypton/argon laser (488-, 568-, and 647-nm excitations are possible) and three photomultiplier tubes with 522DF35, 585EFLP, and 680DF32 emission filters (Table 2), signal intensities of these fluorochromes were measured in polyacrylamide gels containing $\lambda$-DNA. DNA specificity and photobleaching characteristics were estimated from the laser confocal images of the cultured cells [18]. Green fluorescence signals are observed under blue $(488 \mathrm{~nm})$ excitation for nucleic acids stained with BO-PRO-1, YOPRO-1, SYBR Green I, Pico Green, SYTOX Green, and TO-PRO-1. Red fluorescence signals are observed under green $(568 \mathrm{~nm})$ excitation for POPO-3, PI, and YO-PRO3. Far-red fluorescence signals are observed under red $(647 \mathrm{~nm})$ excitation for TOTO-3 and TO-PRO-3. Far-red fluorochromes seem to be particularly useful in three-color imaging in combination with immunofluorescence with fluorescein and rhodamine derivatives [23].

Among the six green fluorochromes, SYTOX Green was DNA-spcific and its fluorescence was relatively intense

Talbe 2. Summary of cytochemical characteristics of nucleic acid-specific fluorochromes.

\begin{tabular}{|c|c|c|c|c|c|c|c|c|}
\hline & $\underset{\mathrm{Ex} / \mathrm{Em}}{\operatorname{maximal}}$ & $\begin{array}{l}\text { excitation } \\
\text { laser } \\
(\mathrm{nm})\end{array}$ & $\begin{array}{c}\text { nuclear } \\
\text { DNA } \\
\text { staining }\end{array}$ & $\begin{array}{c}\text { cytoplasmic } \\
\text { RNA } \\
\text { staining }\end{array}$ & $\begin{array}{l}\text { bleaching } \\
\text { speed }\end{array}$ & $\begin{array}{r}\text { anti- } \\
\text { effe } \\
\text { PPDA }\end{array}$ & $\begin{array}{l}\text { aching } \\
\text { eness } \\
\text { DABCO }\end{array}$ & $\begin{array}{l}\text { total assessment } \\
\text { for cell nuclear } \\
\text { counter-staining }\end{array}$ \\
\hline DAPI $^{1}$ & $358 / 461$ & 351,363 & +++ & - & $\mathrm{NE}$ & $\mathrm{NE}$ & NE & +++ \\
\hline BO-PRO-12 & $462 / 481$ & 488 & + & + & $\mathrm{NE}$ & NE & NE & - \\
\hline YO-PRO-1 ${ }^{2}$ & $491 / 509$ & 488 & +++ & + & + & NE & NE & + \\
\hline SYBR Green $\mathrm{I}^{2}$ & $494 / 521$ & 488 & +++ & - & ++ & ++ & + & +++ \\
\hline Picvo Green ${ }^{2}$ & $502 / 523$ & 488 & ++ & - & ++ & ++ & $+t$ & ++ \\
\hline SYTOX Green ${ }^{2}$ & $504 / 523$ & 488 & +++ & - & + & - & $-*$ & ++ \\
\hline TO-PRO-12 & $515 / 531$ & 488,514 & ++ & + & NE & NE & NE & - \\
\hline POPO-32 & $534 / 570$ & 543,568 & - & +++ & ++ & NE & NE & - \\
\hline $\mathrm{PI}^{1}$ & $535 / 617$ & $543,568^{* *}$ & +++ & ++ & + & NE & NE & + \\
\hline YO-PRO-32 & $612 / 631$ & 568 & $++t$ & - & +++ & +++ & +++ & +++ \\
\hline TOTO-32 & $642 / 660$ & 633,647 & + & ++ & ++ & +++ & +++ & + \\
\hline TO-PRO-32 & $642 / 661$ & 633,647 & +++ & - & +++ & +++ & +++ & $++t$ \\
\hline
\end{tabular}

Results are scored in arbitary units from - to +++ .

maximal Ex/Em: maximal wavelength of excitation/emission.

NE, not examined.

* Bleaching speed of SYTOX Green was weakly accelerated by DABCO.

** PI is also excited under $488 \mathrm{~nm}$ laser irradiation.

1 Reagents purchased from Wako Pure Chemical Industries, Ltd. (Osaka, Japan).

${ }^{2}$ Reagents purchased from Molecular Probes, Inc. (Eugene, OR, USA). 
and stable (Table 2) [18]. SYBR Green I also specifically and intensely stained the cell nuclear DNA, but its fluorescence was bleached rapidly. YO-PRO-1, whose fluorescence was very intense and stable, stained cell nuclear DNA with light co-staining of the cytoplasmic RNA. Therefore, pretreatment of specimens with RNase is recommended for specific nuclear staining with YO-PRO1. BO-PRO-1 stained both the cytoplasmic RNA and nuclear DNA, and when RNase treatment was omitted, it was impossible to distinguish the cell nuclear contour. Among the three red fluorochromes, YO-PRO-3 was DNA specific, and its fluorescence was the strongest (Table 2) [18]. Fluorescence of YO-PRO-3, however, faded rapidly under 568-nm laser irradiation. Although PI was the most stable among the three red fluorochromes, it costained the cytoplasmic RNA. Moreover, PI was also excited by $488-\mathrm{nm}$ laser excitation, and PI fluorescence tended to bleed through the emission filter for green fluorescence. Conversely, POPO-3 preferentially stained the cytoplasmic and nucleolar RNAs, and did not stain the cell nuclear DNA. Of the two far-red fluorochromes, TOPRO-3 specifically and intensely stained the cell nuclear DNA (Table 2) [18, 24], whereas TOTO-3 intensely stained the cytoplasmic and nucleolar RNAs with weak co-staining of cell nuclear DNA. In either case, photobleaching occurred very rapidly.

\section{Prevention of the Photobleaching of DNA-Specific Fluorochromes}

Photobleaching of fluorescent labels is a big problem especially under observation at high magnification. In laser confocal microscopy, illumination by laser beam considerably shortens the fluorescence life of fluorochromes. Prevention of photobleaching is a key for multi-color three-dimensional analysis by laser confocal microscopy, since repeated laser-scanning of the same area accompanied with a slight shift of the Z-axis each time is

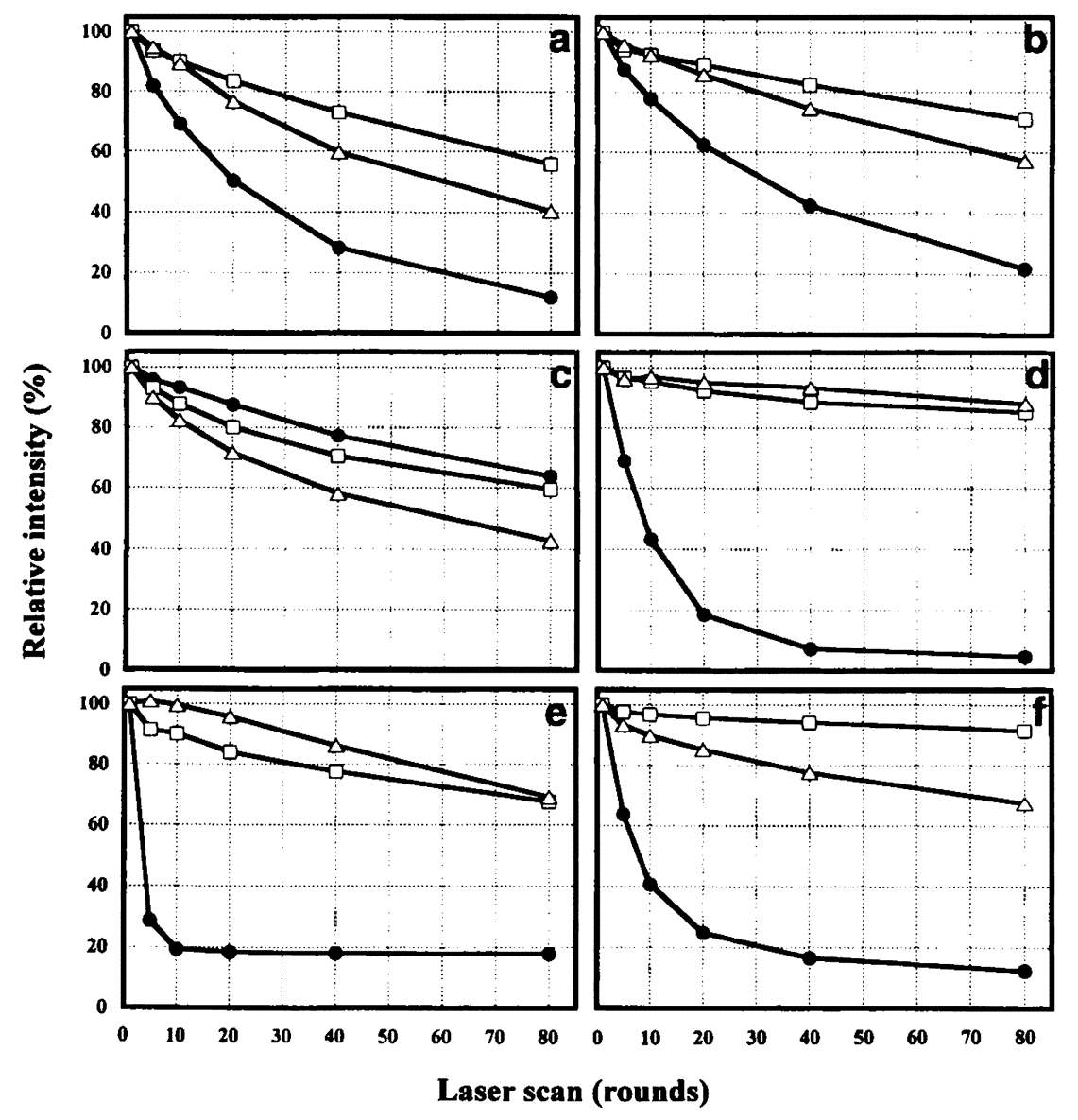

Fig. 1. Effect of anti-bleaching reagents on nucleic acid-specific fluorochromes. MDCK cells were stained with fluorescent dyes, and mounted with a basic mounting medium (control: filled circle), medium containing $0.1 \%$ PPDA (open squre), or 5\% DABCO (open triangle). The fluorescence intensity was measured on a 256-grade scale by the LaserSharp image processing software (Bio-Rad Laboratories, Hercules, CA) in each pixel (a pixel corresponded to $0.211-\mu \mathrm{m}$ square), and the mean intensity of $512 \times 512$ pixels was detemined. The fluorescence intensity was recorded on the designated round of laser scanning. Each round of laser scanning took $1 \mathrm{sec}$, and each pixel in the specimen was illuminated for $3.8 \mu \mathrm{sec}$ per scan. SYBR Green I (a), Pico Green (b), and SYTOX Green (c) were excited at $488 \mathrm{~nm}$. YO-PRO-3 (d) was excited at $568 \mathrm{~nm}$. TO-PRO-3 (e) and TOTO-3 (f) were excited at $647 \mathrm{~nm}$. 
required. TO-PRO-3 seems to be best suited for multicolor analysis, and already has been utilized for the multi-parameter flow cytometric analysis $[6,7,27]$. But, it has one shortcoming: fluorescence instability under laser illumination [18, 24].

Photobleaching of fluorescence is effectively retarded by the addition of anti-bleaching reagents to the mounting medium. PPDA (paraphenylenediamine) and DABCO (1,4-diazabicyclo-(2.2.2)octane) have been successfully used to prolong the immunofluorescence of FITC and rhodamine [28]. We examined the effects of these antibleaching reagents on bleaching characteristic of the following fluorescent dyes: SYTOX Green, SYBR Green I, Pico Green, YO-PRO-3, TO-PRO-3, and TOTO-3 (Fig. 1). PPDA and DABCO (both purchased from Wako Pure Chemical Industries Ltd., Osaka, Japan) were added to the basic mounting media $(90 \%$ glycerol, $100 \mathrm{mM}$ Tris- $\mathrm{HCl}, \mathrm{pH} 8.0$ ) at $0.1 \%$ and $5 \%$, respectively.

Of the green fluorochromes, SYTOX Green was the most stable when anti-bleaching reagents were not used. SYBR Green I and Pico Green also showed good characteristic for nuclear counter-staining except for rapid photobleaching. PPDA and DABCO effectively prolonged the fluorescence of SYBR Green I and Pico Green (Fig. 1a, b, Table 2). Both reagents, however, did not work in SYTOX Green (Fig. 1c, Table 2). Rather, DABCO showed a tendency to accelerate the photobleaching of SYTOX Green. Since anti-bleaching reagents are routinely used for immunofluorescence staining, SYBR Green I seems to be more suitable for cell nuclear counter-staining than SYTOX Green. Of the red fluorochromes, YO-PRO-3 specifically stained cell nuclei. Its excitation and emission peak wavelengths are much longer than those of PI, which effectively reduces possible bleed-through problems. Rapid photobleaching of YO-PRO-3 was overcome by the addition of anti-bleaching reagents (Fig. 1d, Table 2). Photobleaching of TOTO-3 and TO-PRO-3 was also drastically reduced by addition of such reagents (Fig. 1e, f). TO-PRO-3, a DNA specific far-red fluorochrome, seems to be best suited for cell nuclear counter-staining.

\section{v. Conclusions}

SYBR Green I, Pico Green, SYTOX Green (green fluorochromes), YO-PRO-3 (a red fluorochrome), and TO-PRO-3 (a far-red fluorochrome) were found to be suitable for nuclear counter-staining for observation with a laser confocal microsocpe equipped with a krypton/ argon laser tube. Among them, TO-PRO-3 emits farred fluorescence and easily enables three-color fluorescent imaging by combination with immunofluorescence labeling of fluorescein- and rhodamine-labeled antibodies. TO-PRO-3, when used with anti-bleaching reagents, may be best suited for cell nuclear counter-staining (Table 2).

\section{Acknowledgments}

We are grateful to Dr. Keiko Fujikura for technical assistance, and Ms. Shoko Tsukui for secretarial assistance. This work was supported in part by grants-in-aid for scientific reseach from the Ministry of Education, Science, Culture, and Sports of Japan.

\section{References}

1. Brelje, T. C., Wessendorf, M. W. and Sorenson, R. L.: Multicolor laser scanning confocal immunofluorescence microscopy: Practical application and limitations. Methods Cell Biol. 38; 97-181, 1993.

2. Chen, X. -S., Naumann, T. A., Kurre, U., Jenkins, N. A., Copeland, N. G. and Funk, C. D.: cDNA cloning, expression, mutagenesis, intracellular localization, and gene chromosomal assignment of mouse 5-lipoxygenase. J. Biol. Chem. 270; 17993-17999, 1995.

3. De Mazière, A. M., Hage, W. J. and Ubbels, G. A.: A method for staining of cell nuclei in Xenopus laevis embryos with cyanine dyes for whole-mount confocal laser scanning microscopy. J. Histochem. Cytochem. 44; 399-402, 1996.

4. Doornbos, R. M. P., De Grooth, B. G., Kraan, Y. M., Van Der Poel, C. J. and Greve, J.: Visible diode lasers can be used for flow cytometric immunofluorescence and DNA analysis. Cytometry 15; 267-271, 1994.

5. Frey, T.: Detection of bromodeoxyuridine incorporation by alteration of the fluorescence emission from nucleic acid binding dyes using only an argon ion laser. Cytometry 17; 310-318, 1994.

6. Glade, C. P., Van Erp., P.E. J. and Van De Kerkhof, P.C.M.: Epidermal cell DNA content and intermediate filaments keratin 10 and vimentin after treatment of psoriasis with calcipotriol cream once daily, twice daily and in combination with clobetasone 17-butyrate cream or betamethasone 17valerate cream: a comparative flow cytometric study. Br. J. Dermatol. 135; 379-384, 1996.

7. Glade, C. P., Van Erp., P. E. J., Van Hooijdonk, C. A. E. M., Elbers, M. E. and Van De Kerkhof, P.C. M.: Topical treatment of psoriatic plaques with $1 \alpha, 24$ dihydroxyvitamin $\mathrm{D}_{3}$ : A multiparameter flow cytometrical analysis of epidermal growth, differentiation and inflammation. Acta Derm. Venereol. 75; 381-385, 1995.

8. Haugland, R.P.: Handbook of fluorescent probes and research chemicals. 6th ed., Molecular Probes, Eugene, 1996, pp. 144-156.

9. He, P. and Adamson, R. H.: Visualization of endothelial clefts and nuclei in living microvessels with combined reflectance and fluorescence confocal microscopy. Microcirculation 2; 267276, 1995.

10. Hirons, G. T., Fawcett, J. J. and Crissman, H. A.: TOTO and YOYO: New very bright fluorocromes for DNA content analyses by flow cytometry. Cytometry 15; 129-140, 1994.

11. Kahn, E., Hotmar, J. and Bernheim, A.: Three-dimensional fluorescence in situ hybridization. Chromosomal studies on nuclei from cytogenetic preparations by confocal microscopy. Analyt. Quant. Cytol. Histol. 18; 109-114, 1996.

12. Konishi, H., Steinbach, G., Hittelman, W. N., Fujita, K., Lee, J. J., Glober, G. A., Levin, B., Andreeff, M., Goodacre, A. M. and Terry, N.H. A.: Cell kinetic analysis of intact rat colonic crypts by confocal microscopy and immunofluorescence. Gastroenterol. 111; 1493-1500, 1996.

13. Lizard, G., Chignol, M. C., Souchier, C., Schmitt, D. and Chardonnet, Y.: Laser scanning confocal microscopy and quantitative microscopy with a charge coupled device camera 
improve detection of human papillomavirus DNA revealed by fluorescence in situ hybridization. Histochemistry 101; 303310, 1994.

14. Lizard, G., Chignol, M. C., Chardonnet, Y., Soucher, C., Brodes, M., Schmitt, D. and Revillard, J. P.: Detection of human papillomavirus DNA in CaSki and HeLa cells by fluorescent in situ hybridization. J. Immunol. Methods 157; 31-38, 1993.

15. Makowski, E. R. and Ruzin, S. E.: Fluorescent in situ hybridization of maize meiotic chromosomes as visualized by confocal microscopy. BioTechniques 16; 256-263, 1994.

16. Manos, P. and Edmond, J.: Immunofluorescent analysis of creatine kinase in cultured astrocytes by conventional and confocal microscopy: A nuclear localization. J. Comp. Neurol. 326; 273-282, 1992.

17. Marie, D., Vaulot, D. and Partensky, F.: Application of the novel nucleic acid dyes YOYO-1, YO-PRO-1, and Pico Green for flow cytometric analysis of marine prokaryotes. Appl. Environ. Microbiol. 62; 1649-1655, 1996.

18. Matsuzaki, T., Suzuki, T., Fujikura, K. and Takata, K.: Nuclear staining for laser confocal microscopy. Acta Histochem. Cytochem. 30; 309-314, 1997.

19. Nagelhus, T. A., Slupphaug, G., Lindmo, T. and Krokan, H. E.: Cell cycle regulation and subcellular localization of the major human uracil-DNA glycosylase. Exp. Cell Res. 220; 292-297, 1995.

20. Nomura, A., Yamazaki, Y., Tsuji, T., Kawasaki, Y. and Tanaka, S.: Three-dimensional labeling program for elucidation of the geometric properties of biological particles in threedimensional space. Exp. Cell Res. 227; 256-263, 1996.

21. Orsulic, S. and Peifer, M.: A method to stain nuclei of Drosophila for confocal microscopy. BioTechniques 16; 441-
447, 1994.

22. Shellhaas, J. L. and Zuckerman, S. H.: In vitro detection of apoptotic stimuli by use of the HL-60 myeloid leukemic cell line. Clin. Diagn. Lab. Immunol. 2; 598-603, 1995.

23. Suzuki, T., Fujikura, K. and Takata, K.: $\mathrm{Na}^{+}$-dependent glucose transporter SGLT1 is localized in the apical plasma membrane upon completion of tight junction formation in MDCK cells. Hitochem. Cell Biol. 106; 529-533, 1996.

24. Suzuki, T., Fujikura, K., Higashiyama, T. and Takata, K.: DNA staining for fluorescence and laser confocal microscopy. J. Histochem. Cytochem. 45; 49-53, 1997.

25. Takata, K. and Hirano, H.: Use of fluorescein-phalloidin and DAPI as a counterstain for immunofluorescence microscopic studies with semithin frozen sections. Acta Histochem. Cytochem. 23; 679-683, 1990.

26. Takata, K., Kasahara, T., kasahara, M., Ezaki, O. and Hirano, H.: Localization of $\mathrm{Na}^{+}$-dependent active type and erythrocyte/HepG2-type glucose transporters in rat kidney: Immunofluorescence and immunogold study. J. Histochem. Cytochem. 39; 287-298, 1991.

27. Van Hooijdonk, C. A.E.M., Glade, C. P. and Van Erp. P. E. J.: TO-PRO-3 iodide: A novel HeNe laser-excitable DNA stain as an alternative for propidium iodide in multiparameter flow cytometry. Cytometry 17; 185-189, 1994.

28. Valnes, K. and Brandtzaeg, P.: Retardation of immunofluorescence fading during microscopy. J. Histochem. Cytochem. 33; 755-761, 1985.

29. Wright, S. J., Centonze, V. E., Stricker, S. A., De Vries, P. J., Paddock, S. W. and Schatten, G.: Introduction to confocal microscopy and three-dimensional reconstruction. Methods Cell Biol. 38; 1-45, 1993. 